\title{
Radiographic appearance of leptomeningeal disease in patients with EGFR-mutated non-small-cell lung carcinoma treated with tyrosine kinase inhibitors: a case series
}

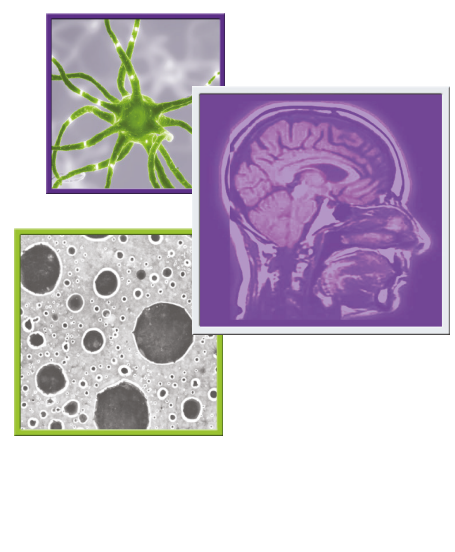

\author{
Ugur Sener*,1, Nassim Matin², Helena Yu³, Andrew Lin'1, T Jonathan Yang ${ }^{4}$ \& Rachna \\ Malani ${ }^{1}$ \\ ${ }^{1}$ Department of Neurology, Memorial Sloan Kettering Cancer Center, New York, NY 10021, USA \\ ${ }^{2}$ Department of Neurology, SUNY Downstate Medical Center, Brooklyn, NY 11203, USA \\ ${ }^{3}$ Department of Thoracic Oncology, Memorial Sloan Kettering Cancer Center, New York, NY 10021, USA \\ ${ }^{4}$ Department of Radiation Oncology, Memorial Sloan Kettering Cancer Center, New York, NY 10021, USA \\ *Author for correspondence: seneru@mskcc.org
}

\section{Practice Points}

- Clinically available tyrosine kinase inhibitors (TKIs) are effective in treatment of EGFR-mutated non-small-cell lung carcinoma (NSCLC).

- Leptomeningeal disease is a known complication of NSCLC with variable manifestations and grim prognosis.

- Targeted treatment with TKIs may achieve partial control of leptomeningeal metastasis from EGFR-mutated NSCLC.

- TKIs may alter the radiographic appearance of leptomeningeal disease, requiring a high index of suspicion and utilization of novel cerebrospinal fluid analysis techniques to establish the diagnosis.

EGFRis frequently mutated in non-small-cell lung carcinomas (NSCLCS). Clinically available tyrosine kinase inhibitors (TKIs) are effective in treating EGFR-mutant NSCLC. In this case series, we present five patients with TKI-treated EGFR-mutated NSCLC who developed leptomeningeal disease (LMD) lacking characteristic imaging findings. All five patients received TKIs prior to development of cytology-confirmed LMD. Clinical signs of LMD preceded radiographic evidence by 2-12 months. T790M, the most common resistance mutation to first-generation EGFR inhibitors, was identified in four cases. These cases illustrate that in patients with EGFR-mutant NSCLC, TKIs may effectively control LMD, creating a lag between onset of symptoms and observation of radiographic findings.

First draft submitted: 20 June 2019; Accepted for publication: 17 September 2019; Published online: 28 November 2019

Keywords: EGFR • leptomeningeal disease $\bullet$ NSCLC • targeted therapy • tumor imaging • tyrosine kinase inhibitors

EGFR is a transmembrane protein involved with cell proliferation, survival and tumorigenesis [1,2]. Driver EGFR mutations have been identified in non-small-cell lung carcinomas (NSCLCs) [3-6]. Deletions in exon 19 and L858R missense mutations in exon 21 are the most common EGFR mutations known to be activating [7,8]. Tyrosine kinase inhibitors (TKIs), such as erlotinib and gefitinib, are effective in treating EGFR-mutant NSCLC as first-line therapy [4,5,8-16]. However, resistance to first-line TKIs develops, with EGFR T790M mutation on exon 20 most commonly detected on repeat biopsy $[15,16]$. Third-generation TKIs, such as osimertinib, have been designed to overcome T790M mutation [15-18].

Leptomeningeal disease (LMD) - defined as spread of cancer to the pia mater, arachnoid mater and the subarachnoid space - is a known complication of NSCLC and carries a poor prognosis, typically if left untreated, in the order of weeks to months [19-22]. Despite advanced imaging and cerebrospinal fluid (CSF) evaluation techniques, establishing the diagnosis can be challenging [19-22]. MRI of the neuroaxis is the imaging modality of choice, but a 
normal MRI does not exclude the diagnosis [19-25]. Tumor cells can be identified with CSF cytology and this remains the gold standard, however, sensitivity of a single sample is low and repeat CSF analyses may be needed [19-22].

Emerging technologies such as cell-free DNA (cf-DNA) and utilizing analysis of CSF circulating tumor cells (CTCs) provide new forms of liquid biopsy. Veridex's (NJ, USA) CellSearch ${ }^{\circledR}$ is a commercially available assay which can be adapted for the detection of CTCs in CSF, based on the expression of a transmembrane glycoprotein called EpCAM detected in tumors of epithelial origin. A benefit of CTC analysis is that it allows for a quantitative evaluation [26-29]. Reported sensitivities of EpCAM-based CSF analysis for detection of LMD has been 76$100 \%$ [26-29]. However, EpCAM is not expressed by all solid organ malignancies and thus CTC technology also has its limitations. In contrast, cf-DNA technology relies on DNA released from neoplastic cells [30-33]. DNA from tumor cells can be detected within the CSF, establishing the diagnosis of LMD. In addition, analysis of CSF cf-DNA can help identify clinically relevant mutations from metastatic and primary nervous system tumors [30-33].

LMD has been described in patients with EGFR-mutated NSCLC. LMD carries a grim prognosis with an estimated survival of less than 6 months despite treatments such as radiation therapy, intrathecal chemotherapy and systemic chemotherapy [19,34]. Emerging data indicates osimertinib penetrates the blood-brain barrier and may represent a therapeutic option after first-generation TKI failure, especially in the setting of T790M mutation [3437]. However, data is limited on the radiographic features of LMD in patients with EGFR mutated TKI-treated NSCLC.

Herein we describe our clinical experience with five patients at Memorial Sloan Kettering Cancer Center (NY, USA) with TKI-treated EGFR-mutated NSCLC who developed LMD while lacking characteristic imaging findings.

\section{Case 1}

A 69-year-old man was diagnosed with EGFR exon 19-deleted adenocarcinoma of the lung in December 2015. Staging MRI of the brain revealed numerous parenchymal brain metastases in January 2016. He was started on erlotinib and had excellent early response with resolution of brain metastases on repeat MRI in June 2016. Worsening pulmonary metastasis in November 2016 led to identification of EGFR T790M mutation, after which he was transitioned to osimertinib.

Clinical signs of LMD developed in October 2016, with the patient reporting vertical diplopia. In November 2016, he developed jaw weakness. MRI brain performed in November 2016, January and February 2017 showed no radiographic evidence of LMD. CSF cytology identified adenocarcinoma in February 2017, confirming clinically suspected LMD. It was only in October 2017 that MRI brain showed scattered evidence of LMD and MRI spine revealed cauda equina involvement (Figure 1).

\section{Case 2}

A 69-year-old woman was diagnosed with EGFR exon 19-deleted adenocarcinoma of the lung in July 2015. Initial staging MRI of the brain revealed multiple brain metastases. Patient had no clinical neurologic signs at the time of diagnosis.

She was treated with whole brain radiation therapy in August 2015, followed by stereotactic radiosurgery to additional metastases the following November. She was initially treated with erlotinib but transitioned to osimertinib in March 2016 when biopsy of a liver metastasis revealed an EGFR T790M mutation.

Clinical signs of LMD developed in spring 2016 with hearing loss noted in the right ear, suggesting involvement of cranial nerve VIII. MRI brain in June and September 2016 showed no radiographic evidence of leptomeningeal metastasis. Lower motor neuron pattern right facial weakness developed in October 2016 and progressed over the following 2 months. Only in December 2016, repeat MRI brain showed ill-defined enhancing lesions along multiple cranial nerves including right cranial nerves VII and VIII in the internal auditory canal (Figure 1). She was treated with skull base radiation in January 2017. Lumbar puncture performed in March 2017 revealed cytology positive for adenocarcinoma, confirming LMD.

\section{Case 3}

A 58-year-old man was diagnosed with EGFR exon 19-deleted adenocarcinoma of the lung in April 2018. At the time of cancer diagnosis, he was found to have a single left posterior midbrain metastasis and associated hydrocephalus, which was treated with a third ventriculostomy and stereotactic radiosurgery. 

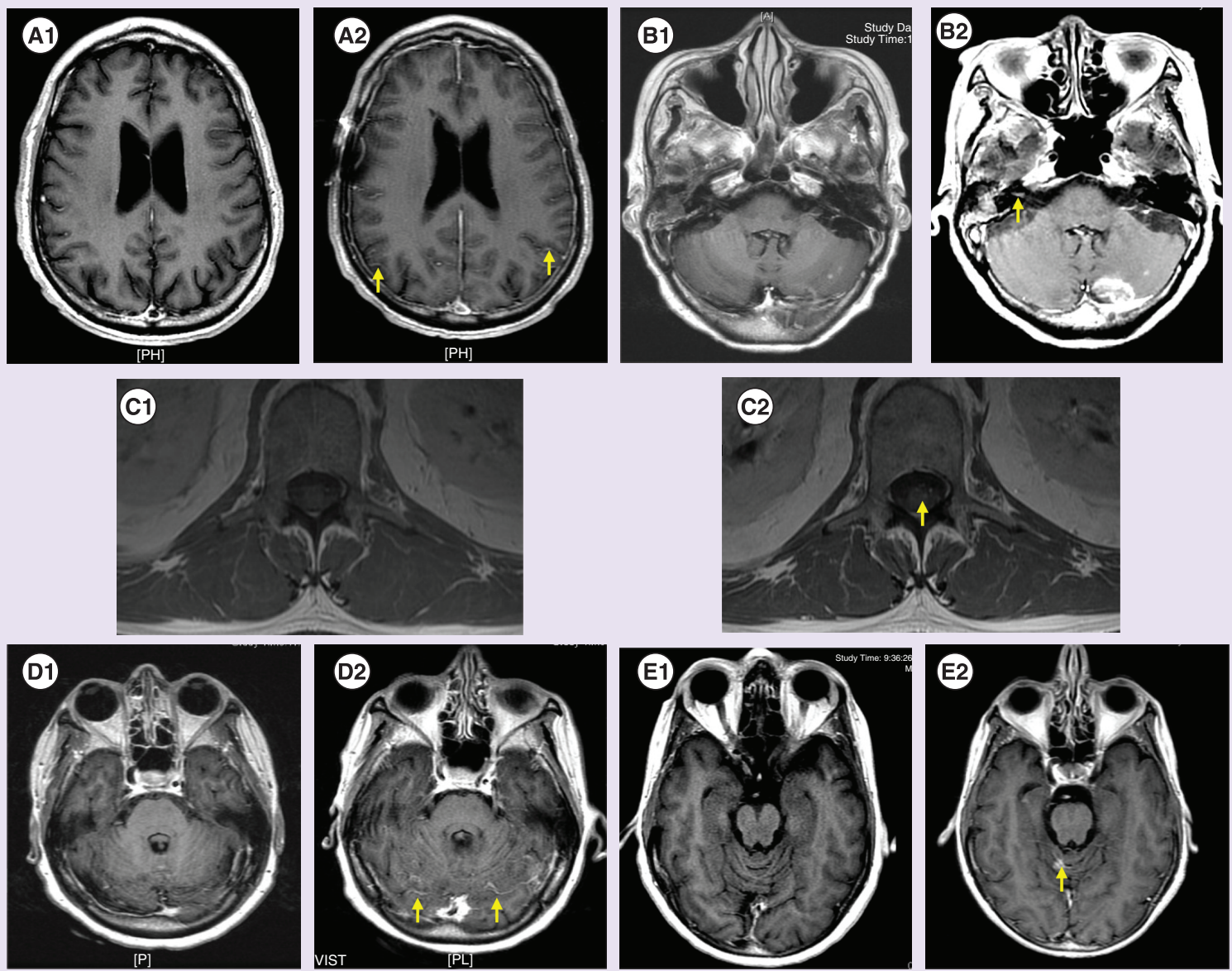

Figure 1. Evolution of radiographic imaging for all cases. (A1 \& A2) Case 1 T1-post contrast MRI brain obtained January 2017 with no evidence of LMD 3 months after onset of vertical diplopia (A1). T1-post contrast MRI brain obtained October 2017 with scattered leptomeningeal enhancement (arrows) as the only radiographic evidence of LMD (A2). (B1 \& B2) Case 2 T1-post contrast MRI brain obtained September 2016 with no evidence of LMD several months after onset of right cranial nerve VII and VIII palsy (B1). T1-post contrast MRI brain obtained December 2016 with enhancement along cranial nerve VII (arrow) providing the only radiographic evidence of LMD (B2). (C1 \& C2) Case 3 T1 post-contrast MRI spine obtained September 2018 with no evidence of LMD (C1). T1 post-contrast MRI spine obtained November 2018 with faint enhancement along conus, providing only radiographic evidence of leptomeningeal disease. D1, D2: Case 4 T1-post contrast MRI brain obtained March 2018 with no evidence of LMD (D1). T1-post contrast MRI brain obtained September 2018 with leptomeningeal enhancement providing first radiographic evidence of LMD (D2). (E1 \& E2) Case 5 T1-post contrast MRI spine obtained September 2017 with no radiographic evidence of LMD (E1). T1-post contrast MRI spine obtained August 2018 with superficial metastasis (arrow) as the only radiographic evidence of LMD (E2).

MRI: Magnetic resonance imaging; LMD: Leptomeningeal disease.

He was started on osimertinib and bevacizumab in May 2018. LMD was clinically suspected in September 2018 when he developed diplopia and confusion, but MRI brain and spine showed no evidence of leptomeningeal involvement. CSF cytology from November 2018 demonstrated suspicious cells concerning for carcinoma. CSF was also analyzed using the CellSearch assay and 40 CTCs were identified, confirming LMD. In November 2018, repeat MRI spine demonstrated faint leptomeningeal enhancement along the conus and cauda equina nerve roots, providing the only radiographic evidence of LMD. Brain MRI remained negative through to February 2019 (Figure 1).

\section{Case 4}

A 60-year-old woman with adenocarcinoma of the lung diagnosed in January 2009 had an EGFR T790M mutation in multiple lung biopsies as well as EGFR L858 mutation in a single left lung nodule. She was initially treated with erlotinib, then transitioned to osimertinib. 
58-year-old man with EGFR exon 19-deleted NSCLC

\section{4/2018:}

Diagnosis of

NSCLC with

single midbrain

metastasis
5/2018:

Started

osimertinib and

bevacizumab
11/2018:

First radiographic

evidence of LMD

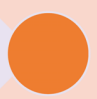

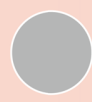

4/2018:

Focal radiation

therapy for

brainstem

metastasis
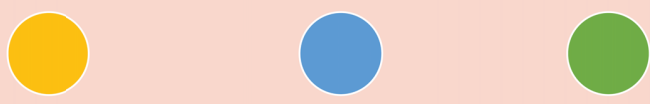

9/2018:

Clinical development

of LMD with diplopia and confusion

Figure 2. Time course from diagnosis of non-small-cell lung carcinoma to radiographic evidence of leptomeningeal disease in Case 3. LMD: Leptomeningeal disease; NSCLC: Non-small-cell lung carcinoma.

LMD was clinically suspected in March 2018 due to persistent vertigo of unclear etiology, but MRI brain and total spine obtained at the time provided no radiographic evidence of leptomeningeal involvement. Patient subsequently developed cognitive difficulty, gait difficulty and incontinence. Lumbar puncture completed in August 2018 revealed cytology positive for adenocarcinoma, confirming the diagnosis of LMD. MRI brain only later in September 2018 demonstrated left cerebellar folia enhancement consistent with leptomeningeal carcinomatosis (Figure 1).

\section{Case 5}

A 75-year-old woman was diagnosed with EGFR L858R mutated adenocarcinoma of the lung in July 2014. Initial staging MRI of the brain revealed multiple parenchymal metastases and she was started on erlotinib. MRI brain in October 2014 demonstrated resolution of previous areas of enhancement. In February 2016, she received focal radiation therapy to an isolated L5-S1 metastasis. In January 2017, she was started on osimertinib due to progression noted in lungs and peripheral blood positive for T790M mutation.

Clinical signs of LMD developed during September 2017 with her reporting severe constipation and urinary retention with overflow incontinence, unexplained by the stable L5-S1 lesion that had been treated with radiation. MRI spine did not show evidence of LMD. MRI brain showed several new parenchymal lesions without definite enhancement. Cytology was negative on lumbar puncture performed in October 2017.

It was only in August 2018 that MRI brain revealed superficial cortical lesions, suggesting radiographic evidence of LMD, and lumbar puncture demonstrated cytology positive for adenocarcinoma (Figure 1).

\section{Discussion}

In this case series we describe five patients with EGFR-mutated NSCLC who developed LMD. All five patients received TKIs prior to development of cytology-confirmed LMD. T790M mutation was identified in four cases. In all cases, clinical signs of LMD preceded radiographic evidence of leptomeningeal involvement by 2-12 months. Figure 2 summarizes representative time course for Case 3.

Observations from this case series have several implications. LMD secondary to EGFR-mutant TKI-treated NSCLC may inherently have minimal radiographic presence, explaining the lack of leptomeningeal enhancement despite presence of clinical symptoms compatible with LMD and positive cytology. On the other hand, use of TKIs may partially control CSF disease, resulting in a paucity of MRI findings. Prior studies have already demonstrated that osimertinib penetrates the blood-brain barrier and may be effective in treating LMD in patients with EGFR T790M-mutated NSCLC, thus improving prognosis [26-28]. In our case series, use of osimertinib may have partially 
controlled LMD, resulting in a paucity of characteristic radiographic features. This hypothesis may be supported in that, patients in our series developed clinical signs 2-12 months prior to radiographic diagnosis. In one patient in our cohort, bevacizumab may have further altered the radiographic appearance of LMD.

The experience with the patients in our case series also underscores the importance of clinically maintaining a high index of suspicion when diagnosing LMD. Given the low sensitivity of traditional testing methodologies, such as serial MRIs and lumbar punctures, there is a need for liquid biopsies. By incorporating novel methods such as CSF CTC detection and cf-DNA analysis into more routine clinical use, we may be able to better detect LMD [26-28]. CTC and cf-DNA analysis can potentially be further leveraged to identify actionable mutations, which can have a direct impact on treatment planning [38,39]. Establishing the diagnosis of LMD and characterizing the nature of tumor involvement in the CSF remains important, with implications on systemic treatment, consideration of intrathecal chemotherapy options and symptomatic management including CSF shunting and radiation therapy.

This case series has several limitations. First, this is a retrospective review of patients evaluated at a tertiary care center. Patients were not controlled for treatments prior to initiation of TKI or dosing of the TKIs administered. One patient received bevacizumab concurrently with TKI treatment. Imaging intervals were not standardized with clinicians obtaining MRIs at their discretion.

Nevertheless, we believe that the observation of a lag between the clinical onset of symptoms and radiographic evidence of LMD in patients with TKI-treated EGFR-mutated NSCLC is a clinically important finding, especially as the lag observed could be up to 12 months. The paucity of radiographic features, negative CSF cytology and the halted neurologic progression is not typical of LMD as described in the literature. The revolutionary impact of TKIs may be transforming LMD into a chronic disease state. We hypothesize neurologic dysfunction without radiographic evidence of LMD may be due to low-level disease activity in these patients with chronic leptomeningeal involvement.

\section{Prior presentation}

Preliminary analysis of the data from this manuscript was presented at the 2019 American Academy of Neurology Annual Meeting in Philadelphia, USA.

\section{Financial \& competing interests disclosure}

This research was supported by a NIH/NCI Cancer Support Grant (P30-CA008748). The authors have no other relevant affiliations or financial involvement with any organization or entity with a financial interest in or financial conflict with the subject matter or materials discussed in the manuscript apart from those disclosed.

No writing assistance was utilized in the production of this manuscript.

\section{Ethical conduct of research}

This project was completed under Memorial Sloan Kettering Cancer Center protocol 17-014 'Patterns of Care, Characteristics, Outcomes, and Quality of Life of Metastatic Patients Treated with Radiation.' This is a retrospective chart review with no experimental intervention performed as part of the study. As such, informed consent was not obtained from included patients, in accordance with regulations of the institutional review board at Memorial Sloan Kettering Cancer Center.

\section{Open access}

This work is licensed under the Attribution-NonCommercial-NoDerivatives 4.0 Unported License. To view a copy of this license, visit http://creativecommons.org/licenses/by-nc-nd/4.0/

\section{References}

Papers of special note have been highlighted as: $\bullet$ of interest; $\bullet \bullet$ of considerable interest

1. Sigismund S, Avanzato D, Lanzetti L. Emerging functions of the EGFR in cancer. Mol. Oncol. 12(1), 3-20 (2018).

2. Jorissen RN, Walker F, Pouliot N, Garrett TP, Ward CW, Burgess AW. Epidermal growth factor receptor: mechanisms of activation and signalling. Exp. Cell Res. 284(1), 31-53 (2003).

3. Rusch V, Klimstra D, Venkatraman E, Pisters PW, Langenfeld J, Dmitrovsky E. Overexpression of the epidermal growth factor receptor and its ligand transforming growth factor alpha is frequent in resectable non-small-cell lung cancer but does not predict tumor progression. Clin. Cancer Res. 3(4), 515-522 (1997).

4. Paez JG, Jänne PA, Lee JC et al. EGFR mutations in lung cancer: correlation with clinical response to gefitinib therapy. Science 304(5676), 1497-1500 (2004). 
5. Lynch TJ, Bell DW, Sordella R et al. Activating mutations in the epidermal growth factor receptor underlying responsiveness of non-small-cell lung cancer to gefitinib. N. Engl. J. Med. 350(21), 2129-2139 (2004).

6. Rosell R, Moran T, Queralt C et al. Screening for epidermal growth factor receptor mutations in lung cancer. N. Engl. J. Med. 361(10), 958-967 (2009).

7. Shigematsu H, Lin L, Takahashi $\mathrm{T}$ et al. Clinical and biological features associated with epidermal growth factor receptor gene mutations in lung cancers. J. Natl Cancer Inst. 97(5), 339-346 (2005).

8. Hsu WH, Yang JC, Mok TS, Loong HH. Overview of current systemic management of EGFR-mutant NSCLC. Ann. Oncol. 29(Suppl. 1), i3-i9 (2018).

9. Shepherd FA, Rodrigues Pereira J, Ciuleanu T et al. Erlotinib in previously treated non-small-cell lung cancer. N. Engl. J. Med. 353(2), 123-132 (2005).

10. Yang CH, Yu CJ, Shih JY et al. Specific EGFR mutations predict treatment outcome of stage IIIB/IV patients with chemotherapy-naive non-small-cell lung cancer receiving first-line gefitinib monotherapy. J. Clin. Oncol. 26(16), 2745-2753 (2008).

11. Rosell R, Carcereny E, Gervais R et al. Erlotinib versus standard chemotherapy as first-line treatment for European patients with advanced EGFR mutation-positive non-small-cell lung cancer (EURTAC): a multicentre, open-label, randomised Phase III trial. Lancet Oncol. 13(3), 239-246 (2012).

12. Zhou C, Wu YL, Chen G et al. Erlotinib versus chemotherapy as first-line treatment for patients with advanced EGFR mutation-positive non-small-cell lung cancer (OPTIMAL, CTONG-0802): a multicentre, open-label, randomised, Phase III study. Lancet Oncol. 12(8), 735-742 (2011).

13. Maemondo M, Inoue A, Kobayashi K et al. Gefitinib or chemotherapy for non-small-cell lung cancer with mutated EGFR. N. Engl. J. Med. 362(25), 2380-2388 (2010).

14. da Cunha Santos G, Shepherd FA, Tsao MS. EGFR mutations and lung cancer. Annu. Rev. Pathol. 6, 49-69 (2011).

15. Oronsky B, Ma P, Reid TR et al. Navigating the "no man's land" of TKI-failed EGFR-mutated non-small-cell lung cancer (NSCLC): a review. Neoplasia 20(1), 92-98 (2017).

16. Liao BC, Lin CC, Lee JH, Yang JC. Optimal management of EGFR-mutant non-small-cell lung cancer with disease progression on first-line tyrosine kinase inhibitor therapy. Lung Cancer 110, 7-13 (2017).

17. Akamatsu H, Katakami N, Okamoto I et al. Osimertinib in Japanese patients with EGFR T790M mutation-positive advanced non-small-cell lung cancer: AURA3 trial. Cancer Sci. 109(6), 1930-1938 (2018).

18. Sequist LV, Soria JC, Goldman JW et al. Rociletinib in EGFR-mutated non-small-cell lung cancer. N. Engl. J. Med. 372(18), 1700-1709 (2015).

19. Nayar G, Ejikeme T, Chongsathidkiet P et al. Leptomeningeal disease: current diagnostic and therapeutic strategies. Oncotarget 8(42), 73312-73328 (2017).

-. Thorough review on diagnosis and management of leptomeningeal metastasis.

20. Pan Z, Yang G, He H et al. Leptomeningeal metastasis from solid tumors: clinical features and its diagnostic implication. Sci. Rep. 8(1), 10445 (2018).

21. Leal T, Chang JE, Mehta M, Robins HI. Leptomeningeal metastasis: challenges in diagnosis and treatment. Curr. Cancer Ther. Rev. 7(4), 319-327 (2011).

22. Le Rhun E, Taillibert S, Chamberlain MC. Carcinomatous meningitis: leptomeningeal metastases in solid tumors. Surg. Neurol. Int. 4(Suppl. 4), S265-S288 (2013).

23. Chamberlain MC, Sandy AD, Press GA. Leptomeningeal metastasis: a comparison of gadolinium-enhanced MR and contrast-enhanced CT of the brain. Neurology 40(3 Pt 1), 435-438 (1990).

24. Schumacher M, Orszagh M. Imaging techniques in neoplastic meningiosis. J. Neurooncol. 38(2-3), 111-120 (1998).

25. Sze G, Soletsky S, Bronen R, Krol G. MR imaging of the cranial meninges with emphasis on contrast enhancement and meningeal carcinomatosis. Am. J. Roentgenol. 153(5), 1039-1049 (1989).

26. van Bussel MTJ, Pluim D, Bol M, Beijnen JH, Schellens JHM, Brandsma D. EpCAM-based assays for epithelial tumor cell detection in cerebrospinal fluid. J. Neurooncol. 137(1), 1-10 (2018).

27. Milojkovic Kerklaan B, Pluim D, Bol M et al. EpCAM-based flow cytometry in cerebrospinal fluid greatly improves diagnostic accuracy of leptomeningeal metastases from epithelial tumors. Neuro Oncol. 18(6), 855-862 (2016).

28. Li YS, Jiang BY, Yang JJ et al. Unique genetic profiles from cerebrospinal fluid cell-free DNA in leptomeningeal metastases of EGFR-mutant non-small-cell lung cancer: a new medium of liquid biopsy. Ann. Oncol. 29(4), 945-952 (2018).

- Demonstrates efficacy of EpCAM-based assay in detection of leptomeningeal metastasis.

29. Boire A, Brandsma D, Brastianos PK et al. Liquid biopsy in central nervous system metastases: a RANO review and proposals for clinical applications. Neuro Oncol. 21(5), 571-584 (2019).

- Perspective on novel approaches to diagnostic evaluation of central nervous system metastases. 
30. Pan W, Gu W, Nagpal S, Gephart MH, Quake SR. Brain tumor mutations detected in cerebral spinal fluid. Clin. Chem. 61(3), 514-522 (2015).

31. Pentsova EI, Shah RH, Tang J et al. Evaluating cancer of the central nervous system through next-generation sequencing of cerebrospinal fluid. J. Clin. Oncol. 34(20), 2404-2415 (2016).

-• Illustrates potential utility of CSF DNA sequencing.

32. Wang Y, Springer S, Zhang M et al. Detection of tumor-derived DNA in cerebrospinal fluid of patients with primary tumors of the brain and spinal cord. Proc. Natl Acad. Sci. USA 112(31), 9704-9709 (2015).

33. Miller AM, Shah RH, Pentsova EI et al. Tracking tumour evolution in glioma through liquid biopsies of cerebrospinal fluid. Nature 565(7741), 654-658 (2019).

34. Park JH, Kim YJ, Lee JO et al. Clinical outcomes of leptomeningeal metastasis in patients with non-small-cell lung cancer in the modern chemotherapy era. Lung Cancer 76(3), 387-392 (2012).

35. Yang JC-H, Kim D-W, Kim S-W et al. Osimertinib activity in patients (pts) with leptomeningeal (LM) disease from non-small-cell lung cancer (NSCLC): updated results from BLOOM, a Phase I study. J. Clin. Oncol. 35(Suppl. 15), 2020 (2016).

36. Saboundji K, Auliac JB, Pérol M et al. Efficacy of osimertinib in EGFR-mutated non-small-cell lung cancer with leptomeningeal metastases pretreated with EGFR-tyrosine kinase inhibitors. Target Oncol. 13(4), 501-507 (2018).

37. Nanjo S, Hata A, Okuda C et al. Standard-dose osimertinib for refractory leptomeningeal metastases in T790M-positive EGFR-mutant non-small-cell lung cancer. Br. J. Cancer 118(1), 32-37 (2018).

38. Jiang BY, Li YS, Guo WB et al. Detection of driver and resistance mutations in leptomeningeal metastases of NSCLC by next-generation sequencing of cerebrospinal fluid circulating tumor cells. Clin. Cancer Res. 23(18), 5480-5488 (2017).

- Demonstrates potential utility of circulating tumor cell analysis for identifying actionable mutations in patients with non-small-cell lung cancer.

39. Ying S, Ke H, Ding Y et al. Unique genomic profiles obtained from cerebrospinal fluid cell-free DNA of non-small-cell lung cancer patients with leptomeningeal metastases. Cancer Biol. Ther. 20(4), 562-570 (2019). 